\title{
Per Marcello Conati
}

\author{
di Gabriele Scaramuzza
}

gabriele.scaramuzza@gmail.com

Fulvio Papi mi ha di recente fatto avere una sua testimonianza, succinta ma densa di memori affetti e di colori, imprescindibile per mettere in luce la rilevanza della figura di Conati nel nostro contesto:

\begin{abstract}
Marcello Conati è stato tra il 1946 e il 1949 il mio compagno di banco al Liceo Classico Carducci. Ci siamo scelti per uno di quei giochi del caso che possono condurre nella direzione di un'esperienza preziosa e quindi di un senso che rinasce nel tempo e non va mai perduto. Ho sempre conservato l'ombra del leggero sorriso di Marcello quando gli chiedevo, ogni volta che lo raggiungevo nella sua abitazione di via Lulli, di suonare al pianoforte la "morte di Isotta". Credo che Marcello con la sua silenziosa discrezione conoscesse i miei fragili segreti meglio di me. Aveva due anni in più passati al Conservatorio: l'amicizia spontanea, la solidarietà e certo il filo di affetto avevano forse una radice in questo dislivello allora importante, del tutto privo di retorica e, anzi, capace di un affetto pedagogico rispetto al mio lessico eccessivo e forse un poco conformista. Nasceva così una mia taciturna ammirazione. Ci siamo persi di vista nei primi anni Sessanta quando sentimenti, abilità, ancora oblique vocazioni e una certa immaginazione conducevano per i primi sentieri della nostra vita. La contesa con il tempo non si può che perdere. Ma la memoria, anche senza graffio ha la sua dolorosa rivincita. Così anche se studiassi con nuova volontà il prezioso saggio di Scaramuzza su Marcello, restava soprattutto indimenticabile quel suo mezzo sorriso. Marcello era diventato un celebre studioso verdiano: non era una strada che ero in grado di percorrere. Restava la sua affettuosa condiscendenza liceale che non mi pare di aver avuto, se non raramente, per la mia fantasia.
\end{abstract}

Non è dunque solo per il suo rilievo cultural-musicale che vogliamo ricordare qui Conati, mancato nel dicembre del 20181; né solo perché è nato a Milano nel 1928 e nella nostra città si è formato. Ma anche perché qui, al Liceo Classico Carducci, da compagno di banco di Fulvio Papi, ha respirato un'aria in certo modo affine alla nostra. Di Conati Papi mi ha sempre parlato con ammirazione e affetto; mi ha confessato anzi di dovere a lui qualcosa del suo amore, sia pur da mero ascoltatore, verso il mondo musicale; ricorda con

\footnotetext{
${ }^{1}$ Paolo Isotta gli ha dedicato un bel ricordo ne «Il Fatto Quotidiano» del 24 marzo 2019.
} 
nostalgia che gli suonava al piano musica classica, talvolta (come s'è visto) il celebre Isoldens Liebes-Tod. Certo non Verdi, e questo non è privo di significato come vedremo.

Diplomato in pianoforte, composizione e direzione d'orchestra, Conati non ha svolto le sue principali, molteplici attività a Milano: musicologo e musicista riconosciuto, è stato maestro sostituto all'Operahaus di Zurigo, poi docente di arte scenica al Conservatorio di Parma - città in cui ha collaborato anche con l'Istituto Nazionale di Studi Verdiani, contribuendo anche alla pubblicazione nel 2018 del Carteggio Verdi-Boito.

La mia innata simpatia per Verdi mi ha indotto un grande interesse verso tutte le sue molteplici e ricche ricerche verdiane. Soprattutto perché mi hanno offerto, oltre a conoscenze indispensabili, un riconoscimento qualificato, e atteso, della mia esperienza di mero spettatore. Appassionata, certo, ma imparagonabile con quella di Conati, infinitamente più esperta sullo specifico piano drammaturgico-musicale e culturale.

Sicuramente la mia scoperta di Conati è avvenuta leggendo la riedizione da lui curata dell'Autobiografa dalle lettere, di Verdi ${ }^{2}$. Non sono da dimenticare poi le laboriose e dettagliate ricerche che lo hanno condotto a $L a$ bottega della musica, Verdi e La Fenice ${ }^{3}$, e soprattutto al bellissimo Verdi. Interviste e incontrit ${ }^{4}$. Costante è la sua acribia, la sua cura del particolare, il suo rifiuto di disperdersi in considerazioni generiche o, per altro verso, in un'erudizione fine a sé. Non a caso, nel suo tuttora insostituibile Giuseppe

\footnotetext{
${ }^{2}$ Nel 1981 è uscita la nuova edizione, interamente rivista, con annotazioni e aggiunte di Conati, di Giuseppe Verdi: Autobiografa dalle lettere, a cura di A. Oberdorfer, Rizzoli, Milano 1981 (la prima edizione è stata del 1941, la seconda del 1951).

${ }^{3}$ Edito da Il Saggiatore, Milano 1983.

${ }^{4}$ La seconda edizione (corretta, aggiornata e accresciuta non poco) è uscita presso EDT, Torino 2000. La prima edizione, sempre curata da Conati, è uscita da Il Formichiere a Foligno nel 1980.
} 
Verdi. Guida alla vita e alle opere ${ }^{5}$, Conati ci offre percorsi che attraversano insieme i contesti teatrale, musicale ed esistenziale di Verdi; e il tutto è accompagnato da una documentazione adeguata, che non trascura le immagini, le dichiarazioni di poetica, i contesti storici e culturali.

Di persona ho incontrato Conati una sola volta, a Parma, a quanto ricordo. Ma ho sempre letto con partecipazione i suoi lavori. Gli ho inviato qualche mio scritto, ricevendone pareri comunque per me giovevoli, soprattutto quando critici; mi è stato riconoscente della mia recensione ${ }^{6}$ di Piegare la nota. Ed è stata proprio la pubblicazione di questo libro 7 a offrirmi l'occasione, oltre che di segnalare un importante contributo agli studi verdiani, di dedicare al suo autore, Marcello Conati appunto, qualche nota che da tempo avevo in animo.

Di grande rilevanza è stata per me la modalità dell'approccio di Conati a Verdi, rovesciato rispetto a quello solito: che tende a relegare l'amore per Verdi (se mai c'è stato) in una sorta di peccato giovanile, da cui riscatta un più maturo approccio al mondo musicale (wagneriano). Nell'introduzione a Piegare la nota scrive Conati: «allevato da mio padre, cantante, al culto di Wagner», a «capire Verdi ci sono arrivato piuttosto tardi», e «non certo per

\footnotetext{
5 ETS, Pisa 2002. Aggiungo a latere che nel 2014 è uscito, di Conati, "Si può dire una menzogna in musica?", in A. Calzolari (a cura di), Mondobugia. Undici variazioni sul mentire, Mimesis, Milano 2014. Interessante quanto Conati annota: ci può essere la menzogna nel teatro drammatico tutto (e qui le esemplificazioni non mancano, da Mozart a Rossini, da Puccini a Verdi), «è solo attraverso le parole del libretto e l'azione scenica che la musica può esser coinvolta, suo malgrado, in un mendacio. Per sua natura, per le leggi stesse che la governano [...] non le è consentito d'essere altro che se stessa».

${ }^{6}$ G. Scaramuzza, "Il Verdi di Marcello Conati" in Odissea, maggio 2015.

7 M. Conati, Piegare la nota. Contrappunto e dramma in Verdi, Olschki, Firenze 2014; libro che cade nel contesto della rammemorazione dei duecento anni dalla nascita di Verdi. Se si vuole avere un quadro più completo della bibliografia verdiana di tempi più o meno coevi, è necessario tener presente quanto meno lo studio esauriente di Raffaele Mellace, Con moltissima Passione. Ritratto di Giuseppe Verdi, Carocci, Roma 2013; e anche, dello stesso autore, "Eredità di un centenario: Verdi e il suo mondo in due ambiziosi lavori di sintesi", Studi Verdiani, XXIV, 2014, pp. 270-280; in questo caso di un vero e proprio saggio si tratta, anche se prende l'avvio da una - pur ricca e ragionata comunque - recensione critica di Verdi Handbuch e di The Cambridge Verdi Encyclopedia (entrambi del 2013).
} 
folgorazione», bensì "per gradi», attraverso una conoscenza pratica e un sistematico approfondimento dei problemi del suo teatro.

Nella lunga esperienza di musica e di vita di Conati Verdi è stato una conquista raggiunta con grande dedizione, un traguardo. Questo è tanto più rimarchevole se si considera che proprio da Wagner è partito; non è approdato alle rarefatte atmosfere wagneriane lasciandosi alle spalle scontate (considerate persino riprovevoli poi) inclinazioni giovanili, emancipandosi da iniziali e troppo "facili" simpatie verdiane. Tanto meno ha fatto agire Wagner come metro di giudizio cui tutto commisurare, né il suo teatro come modello insuperabile in base a cui considerare, giustiziare anzi, tutto il teatro musicale. Se gli è ovviamente estraneo ogni verdismo corrivo, non di rado davvero imbarazzante, è ben lontano - e questo resta per me decisivo - «da tutti coloro che, assumendo un atteggiamento di aristocraticità estetizzante, prendono partito in favore di Wagner e disprezzano il teatro verdiano»8.

Il mio personale cammino dentro Verdi è stato l'opposto di quello di Conati, assai diverso è stato il mio approccio: da mero per quanto attento ascoltatore; non da musicista né da musicologo. Posso rintracciare le date del nascere della mia passione per Bach, Mozart, Beethoven, Mahler, Stravinskij o Webern. Ma non quelle del nascere del mio amore per Verdi, che mi è dentro da sempre, come una sorta di dato di natura strettamente intrecciato al mio vissuto. Non sarò mai abbastanza grato a musicologi e uomini di cultura, non numerosissimi in verità, dotati di sensibilità in grado di apprezzare Verdi, e di cogliere da competenti la sostanza della sua opera. Vorrei citare qui innanzitutto, insieme a Conati, Massimo Mila, Fabrizio Della Seta, Julian Budden, e poi va via Gilles De Van, Pierluigi Petrobelli, Emilio Sala...

Conati non ha mai recluso Verdi nell'immagine, pur giustificata, ma di per sé inadeguata, dell'uomo di teatro; «poco a poco, analisi dopo analisi» ha

\footnotetext{
${ }^{8}$ Mutuo queste parole dall'articolo di A. Mazzoni: "Da Gramsci a Parinetto. Il melodramma italiano: popolo e rivoluzione", apparso su «BresciaMusica».
} 
saputo coglier la grandezza di Verdi anche come musicista. Cosa che si vede in modo esemplare nel suo Rigoletto ${ }^{9}$, dove quest'opera è affrontata con un ampio respiro culturale, oltre che con specifica competenza drammaturgicomusicale.

Tanto meno tacerei le sue ricerche su una che considero tra le migliori in assoluto delle opere verdiane: Simon Boccanegra, del quale nel 1993, presso Ricordi, ha curato la Disposizione scenica. Accanto ad altri scritti, una "ricognizione", la sesta di Piegare la nota, è specificamente dedicata a questo dramma. Tensioni utopiche non sono assenti da quest'opera; basti pensare all'anelito verso la pace più volte espresso (anche se sempre contraddetto dai fatti) da Simone; basti pensare allo svolgersi della vicenda, al finale, di cui Emilio Sala in una sua bella conferenza nel Ridotto della Scala ha sottolineato l'apertura alla speranza; nessuna opera di Verdi può propriamente esser detta pessimista, mantenendo nella sua "tinta" musicale toni di utopica speranza.

Nel complesso, il libro Piegare la nota consiste in una raccolta di saggi apparsi tra il 1969 e il 2007, dedicati a creazioni verdiane diverse: da Oberto Conte di San Bonifacio, Macbeth, Stiffelio, Luisa Miller, I Vespri siciliani, Aida, fino all'Ave Maria. Tutti i saggi sono tuttavia riuniti sotto un indice comune: il contrappunto nei drammi verdiani, come indica anche il titolo.

\footnotetext{
9 M. Conati, Rigoletto. Un'analisi drammatico-musicale, Marsilio, Venezia, 1992 (prima edizione Mondadori 1983); con dedica a suo padre, significativa nel nostro contesto. A p. IX leggiamo: «la drammaturgia di tutto il teatro verdiano trova il suo primo fondamento nella musica, o meglio nella scelta e nell'organizzazione di dimensioni sonore atte a esprimere il dramma nei suoi più diversi aspetti onde coinvolgere lo spettatore e suscitarne l'emozione. Per Verdi è la musica che deve possedere il dramma, non viceversa. Per questa via mi è stato possibile superare quella sorta di diffidenza che un tempo nutrivo per i capolavori più popolari di questo compositore e comprenderne sempre meglio i valori teatrali attraverso la loro espressione musicale. E sempre più chiara mi è parsa una qualità somma di Verdi: il suo magistrale dominio dei mezzi musicali, quella straordinaria facoltà che gli ha consentito di affrontare il dramma in tutte le sue componenti e di esprimerne - per contrasto o per transizione, per accostamento o per sovrapposizione - la commedia e la tragedia».
} 
Scrive Conati nell'introduzione: «Fatti i conti con quanto da me pubblicato in cinquant'anni di ricerche sulla vita e sull'opera di Verdi, la scelta definitiva degli argomenti è stata ispirata dal proposito di dare ampio spazio all'arte di Verdi e alla sua poetica di architetto in musica, ponendo in luce i suoi processi compositivi in funzione drammaturgica, esaminando i criteri di alcuni suoi rifacimenti, trattando di fonti drammatiche, di messinscena, di ballabili, di musica popolare, di problemi storiografici, di contrappunto...». Nella consapevolezza che "è nel suo magistero musicale, costruito pietra su pietra alla scuola "napoletana" di Vincenzo Lavigna, che affonda le radici, a parer mio, il suo straordinario senso del teatro». Sempre Conati sottolinea «limportanza decisiva esercitata sulla formazione musicale» di Verdi «dall'apprendimento tenace, quasi ossessivo, del contrappunto e dello stile fugato». Opportunamente riprende nel Preambolo al suo libro noti passi verdiani, ne cito uno per tutti: «per fare un'opera bisogna aver in corpo primieramente della musica»; e Conati aggiunge a chiarimento: «l'interesse che in Verdi suscita un soggetto d'opera prende sempre le mosse dalla possibilità di rinvenirvi le occasioni atte a tradurre gli effetti drammatici in strutture musicali in movimento, compiute e autosufficienti». Verdi è attratto insomma innanzitutto, più che dai contenuti, dalla musicabilità di un testo.

Verdi ama presentarsi come un artista istintivo e spontaneo, ma ha alle spalle una solida formazione musicale. Dichiara (in un passo che Conati cita) di essere «il meno erudito di tutti" i musicisti passati e presenti». Ma aggiunge: «dico erudizione e non sapere musicale. Mentirei se dicessi che nella mia gioventù non abbia fatto lunghi e severi studj. Egli è per questo, che mi trovo aver la mano abbastanza forte a piegare la nota come desidero. Ed abbastanza sicura per ottenere, ordinariamente, gli effetti ch'io immagino». Non a caso Conati dà rilievo al "coraggioso sperimentalismo" di Verdi, che porta fino alle soglie del Novecento.

Tra le intuizioni di Conati che più incoraggiano le mie impressioni di ascolto c'è quella della funzione drammaturgica e non esornativa dei ballabili: 
"i ritmi di danza sono spesso sfruttati da Verdi in funzione drammatica: essi vengono inseriti nel corso dell'azione scenica per definire musicalmente una situazione" (leggiamo nella quinta "ricognizione", dedicata ai ballabili dei Vespri siciliani). Così gli esotismi di Aida, in relazione ai quali Conati riprende osservazioni assai penetranti di Della Seta (per cui l'aspetto esotico non è «un fatto di gusto e di colore», ma un «elemento stilistico» rilevante drammaturgicamente). Qualcosa di analogo, direi (è una mia aggiunta), si può dire dei brindisi, diffusi nell'universo operistico verdiano - basti pensare anche solo a quello del Macbeth. Ma anche, in particolare, della «musica cupa e lugubre» che «annuncia l'arrivo di Fenena e degli Ebrei condannati a morte» nel quarto atto di Nabucco, e della «musica villereccia» che annuncia l'arrivo di Re Duncano nel primo atto di Macbeth, Macbeth (su quest'ultima non dimenticherei le osservazioni di Francesco Degrada nel programma di sala della Scala del 1997-1998), entrambe con un andamento vagamente bandistico ma intensamente espressivo.

A testimonianza del respiro ampio, accurato ma mai erudito fine a sé, degli scritti di Conati, sono rimasto colpito da talune sue notazioni sulla Messa $d a$ Requiem, che danno rilievo al sapore profondamente esistenziale che la intride ${ }^{10}$.

Tra i tanti scritti sul Requiem verdiano segnalo per la sua densità drammatica, di Josef Bor, Il Requiem di Terezin ${ }^{11}$, che parla della sua

10 In un'ottica personale simili notazioni si associano a pagine che le ha dedicato Alice Cappagli. Nel cui saggio Variazione eidetica dell'opera musicale. Il Requiem di Verdi, scrive: la Messa da Requiem, al «carattere mistico del rito», aggiunge «il senso ultimo e comune all'umanità dell'incontro con la morte e quindi col mistero, con il nulla e con un'eternità negativa non ancora esperita e tuttavia inevitabile». Non solo "tocca con drammaticità il senso della morte ma, cosa propria del misticismo, tocca quello proprio della "morte eterna", del nulla che si sovrappone alla notte, che si contrappone a quello di una "vita eterna" oggetto esclusivo della fede. Tutto il testo, dall'inizio alla fine è pervaso da timore, attesa, speranza, tanto che il Dies irae torna a irrompere alla fine nel Libera me Domine con il suo non a caso "allegro agitato", che spezza l'implorazione dopo una sospensione di silenzio generale in cui si spegne un "pianissimo, allargando e morendo"». A. Cappagli, Variazione eidetica dell'opera musicale, Materiali di Estetica. Nuova serie, I, 2010, pp. 71-79.

${ }_{11}$ Tr. it. di Bruno Meriggi, Passigli, Firenze 2014. 
preparazione ed esecuzione nel lager di Terezin. Inutile dire il profondo afflato utopico che percorre anche solo il semplice progetto di una simile iniziativa in uno tra i più terribili centri di deportazione degli ebrei durante le Seconda guerra mondiale.

Conati della Messa mette in luce il "sapore terrestre» che promana dalla «materialità stessa dell'esistenza umana espressa attraverso il canto». Tutto il teatro verdiano, «popolato di infelici, di perseguitati, di "diversi", di vittime sacrificali, sembra rianimarsi attraverso le inquiete pagine della Messa. Il gesto teatrale viene interamente assorbito dalla rappresentazione musicale del terrore dell'uomo serrato in un inesorabile confronto con la propria natura, della sua ribellione di fronte alla morte, del suo sgomento alla soglia dell'eterno ignoto, attraverso un percorso in cui l'accento epico s'alterna a quello elegiaco, il terrore allo sconforto, la preghiera allo scatto iracondo, il grido della disperazione a quell'anelito alla vita, che è il Libera me Domine».

Non poco di tutto questo, mutatis mutandis, è utile per meglio comprendere i toni esistenziali e utopici ben presenti in non poche altre opere di Verdi; di un'atmosfera metafisica, aggiungo è pervasa persino la sua opera considerata più melodrammatica, Il Trovatore. Le ricerche di Conati non mirano solo a dare il giusto rilievo alla sapienza costruttiva, tecnico-artistica, di Verdi; né al suo mero ruolo storico-artistico. Tendono soprattutto a riscattare, mediante questo, lo spessore estetico-esistenziale delle sue opere. Non a caso, sempre nell'introduzione a Piegare la nota, Conati annota che a Verdi è giunto per il fascino che ha esercitato su di lui l'uomo Verdi - che è anche il titolo del noto libro di Frank Walker. Se così non fosse ogni sua fatica sarebbe stata ben poco remunerativa sul piano della sua vita. Il valore di un'opera non è mai solo artistico: è insieme sempre anche esistenziale. Investe insieme ciò che si dice "l'uomo" e insieme l'“opera". 